\title{
LES ASPECTS MARITIMES \\ DE LA POLITIQUE ITALIENNE D'ALPHONSE V D'ARAGON; problèmes techniques: les types de navires
}

\section{Georges Peyronnet}

\section{Introduction}

La réussite de la politique italienne d'Alphonse V, aboutissant de 1420 à 1443 à la conquête du royaume de Naples, nécessita des efforts prolongés et coûteux sur terre et sur mer. Nous voudrions ici souligner quelques aspects matériels de cette action qui fut menée de façon méthodique et tenace, et l'on insistera sur ce qui concerne l'histoire maritime. Nous avons dégagé plusieurs directions de recherches à partir des dépouillements que nous avons effectués dans les dépôts d'archives italiennes, surtout aux Archives d'Etat de Venise et de Gênes, ainsi que dans les dépôts de Barcelone (cf. supra). Du matériel ainsi rassemblé, l'on peut tirer des indications dans les domaines suivants: technique nautique de l'époque (types de navires, tonnage, effectifs, équipement, conditions de navigation), commerce par mer, piraterie, droit maritime. Nous nous bornerons ici, vu l'espace dont nous disposons, au premier point: les types de navires; c'est un sujet encore mal connu, controversé, sur lequel il convient d'apporter quelque lumière. 
Il serait bon de décrire d'abord les types de navires principaux de la flotte catalane au début du xvème. siècle'. Rappelons qu'il n'y a guère de différence à cette époque entre bâtiments marchands et militaires. Tous les vaisseaux servent en principe au commerce (excepté les galères légères de Venise exclusivement militaires), mais ils peuvent également jouer un rôle défensif ou pratiquer la course: soit isolément soit en groupes plus ou moins cohérents. Les marins, hommes libres salariés, avaient toujours des armes offensives et défensives. On disait d'un navire qu'il était «armé en guerre» quand il adoptait certaines dispositions techniques en vue du combat (matelassage de cuir, palissades de bois sur les bastingages...) et, mieux encore, lorsque ce navire embarquait des soldats en plus de l'équipage, notamment des archers ou des arbalétriers. Mais à part ces détails, les types de navires étaient les mêmes en paix comme en guerre.

Pour ce qui concerne la flotte catalane, ces types peuvent se ramener essentiellement à trois. La galère (en catalan galea, parfois galere ou galee) était le bâtiment méditerranéen par excellence, à voile et à rame; c'était le type de vaisseau qui assurait la majeure partie du commerce maritime catalan: le type le plus rapide et le plus apte au combat ${ }^{2}$. La nef (nau ou nave) marchait essentiellement à la voile, car c'était un navire de haut bord et plus large; ordinairement destinée au transport des marchandises, elle pouvait servir de transport de troupes dans une expédition militaire: elle portait alors au maximum 500 hommes ou 150 chevaux; les plus importantes avaient trois ponts et jaugeaient dans les 450 à 500 tonnes: approximation basée sur la charge de la nef en salmas de blé, chaque salma valant environ 2 hectolitres, soit $150 \mathrm{~kg}$. Enfin le leny (en catalan «bois», du latin lig-

${ }^{1}$ Sur les difficultés de cette étude, $c f$. DufourcQ, Charles-Emmanuel, L'Espagne catalane et le Maghreb aux XIIlème et XIVème siècles, Paris 1966. pp. 36-53.

2 CARRERE, Claude, kLe droit d'ancrage et le mouvement du port de Barcelone au milieu du Xvème siècle», dans Estudios de Historia Moderna, Barcelona 153 , pp. 93-96. 
num) était une embarcation légère, de forme et de tonnage variables: entre 25 et 125 tonnes; il pouvait marcher à la rame seule; robuste, stable et rapide à la fois, ses qualités le rendaient tout à fait apte à naviguer en Méditerranée: c'est pourquoi c'était la catégorie de navires la plus répandue. Tenons compte cependant que les limites entre ces variétés de bâtiments demeuraient incertaines. Une idée de la diversité des navires est donnée par le fait que le leny le plus petit portait 4 à 5 matelots, tandis que le plus grand était conduit par 80 à 100 rameurs; à l'autre bout de l'échelle, une grande galère était montée par 100 à 180 hommes, dont plus de 100 rameurs. On rencontre aussi le terme fusta (au sens propre: bois de charpente) qui s'applique à tous les types de bâtiments, et le mot barca (ou barcha) qui peut représenter un leny moyen.

L'on reste gêné par l'imprécision du vocabulaire, car dans les documents en latin navis, terme classique, est employé pour «nef» aussi bien que pour «galère», la nef étant un type de navire inconnu de l'Antiquité. Certes, on trouve précisé pour la galère soit navis longa, soit triremis: mais ce n'est pas une règle toujours respectée ${ }^{3}$. Quoi qu'il en soit, la composition des convois dont se servit Alphonse V visait sans nul doute à faire protéger les nefs par les galères. En effet celles-ci, ne dépendant pas du vent, manoeuvraient plus librement pour prévenir un combat, pour l'engager, ou pour le mener à bien. La tactique maritime du Magnanime correspond à sa politique: il ne s'interesse pas à la guerre de course; il organise des convois lourds de transports d'hommes, de chevaux, d'engins et de vivres afin de poursuivre des opérations terrestres de longue durée. La protection par les galères est toutefois relativement importante: elle ne sert pas seulement d'escorte, elle forme également une masse de manoeuvre contre une escadre adverse.

${ }^{3}$ Cette imprécision du vocabulaire ne permet pas d'apprécier pleinement la remarque de M. Jacques HEERs notant que vers 1460 a Barcelone on ne voit "que des galées ou des navires minuscules) (Le Navire et l'Economie maritime du Moyen Age au Xvrtème siècle, principalement en Méditerranée, Paris 1958, pp. 109-110). Vers 1460 en effet, la paix avait été rétablie entre Gênes 
En fonction des données que nous venons d'exposer, nous commencerons notre étude par les nefs; ensuite nous traiterons des galères, puis des autres types de bâtiments.

\section{Les nefs}

Les nefs catalanes d'Alphonse V sont le plus souvent armées, c'est-à-dire munies d'artillerie ${ }^{4}$; en outre elles portent quelques centaines de gens d'armes et des arbalétriers; une nef «bien arméen (be armada) porte des cavaliers (octobre 1422). Pour le tonnage, il est d'un minimum de 600 botes par bâtiment pour 3 ou 4 nefs «très bien armées» qu'Alphonse V demande à sa femme de lui préparer en août 1423. Et dès le mois de décembre 1422 le roi d'Aragon donnait l'ordre à son vice-roi de Sicile de faire construire deux nefs de 1000 botes chacune. Mais l'estimation s'avère d'autant plus difficile qu'il a été jusqu'à présent impossible de trouver l'équivalent métrique de la bota de Barcelone. La botta de Naples approchait au moins 470 litres'. Nous

et Jean II d'Aragon: celui-ci n'avait donc plus besoin de nefs à usage militaire, au contraire de son frère Alphonse $\mathrm{V}$.

${ }^{4}$ Bien que les sources catalanes des xivème et xvème siècles confondent, pour l'artillerie portée, les nefs avec les galères, on peut se baser sur elles pour noter que les nefs portaient des bombardes dès 1369. C'est la conclusion tirée par CAPMANY, Antonio de, Memorias históricas sobre la marina, comercio $y$ artes de la antigua ciudad de Barcelona, t. IV, Madrid 1792, et par SALAS, Francisco Javier de, "Galeras de los siglos XV y XVI) (dans Museo Espaniol de Antigüedades, t. VII, Madrid 1876, pp. 541-543).

${ }^{5}$ Si l'on considère la botta di mena napolitaine, à Napples même il en existait deux, l'une valant un peu plus de 500 litres, et l'autre environ le double (HeErs Jacques, Le livre de comptes de Giovanni Piccamiglio, homme d'affaires génois, 1456-1459, Paris 1959, p. 23 n. 2). L'expression bota de mena existait également en Catalogne, où elle désignait un tonneau pour contenir du vin, moins grand qu'un bocoi, qui pouvait valoir plusiers centaines de litres (Alcover, Diccionari català-valencià-balear, art. Mena, t. VII, Palma de Majorque 1956, p. $338 \mathrm{col}$. 1); les Catalans employaient aussi l'expression bota 
savons que celle de Barcelone valait 10 quintas ${ }^{6}$. Mais que représentait la quinta? Peut-être faut-il la rapprocher du «quintal» catalan actuel de $40 \mathrm{~kg}$, voisin du «quintal» marseillais d'environ $38 \mathrm{~kg}$ utilisé du xinème au xvmème siècle, et du cantar de Gênes de 47,6 kg qui servait à jauger les lourdes nefs génoises porteuses d'alun. La bota barcelonaise équivaudrait alors à peu près à $400 \mathrm{~kg}$ de port'.

On peut penser que les Catalans utilisaient la bota comme unité de poids et non de capacité, de la même façon que les Génois employaient le cantar. Une preuve de la confusion médiévale entre la jauge et le port est l'emploi par les Génois d'une autre unité de mesure, la vegeta, qui, pour les navires, valait environ 500 litres, soit à peu près une botta di mena. Mais en donnant un sauf-conduit à un Génois pour la navis de ce dernier en 1444, Alphonse $\mathrm{V}$ signale pour de bâtiment portatus vegetum quadringentarum vel inde circa, soi environ 400 vegete de port: on hésite alors, sur la base de l'équivalence entre 1 botta, 1 vegeta et 10 cantari, entre 60 tonneaux de jauge et 160 tonnes de port. Et l'on retombe dans une autre confusion à propos du terme navis, qui veut dire ici simplement «navire» sans que l'on puisse autrement préciser, car les chiffres obtenus sont trop faibles pour une nef, surtout génoise.

maresa pour désigner un tonneau de vin bien préparé (ALCOVER, id., art. Marès. 2, ibid., p. $240 \mathrm{col}$. 1). Mais l'estimation précise variait suivant les ports, et même selon le patron du navire.

${ }^{6}$ DEL TREPPO, Mario, "Assicurazioni e commercio internazionale a Barcellona nel 1428-1429», Rivista Storica Italiana, a. LXIX, 1957, p. 257. De même, à Gênes, la botta (de Naples) valait 10 cantari (HEERS J., op. cit., p. 23; id., Gênes au Xvème siècle, Paris 1961, p. 269).

${ }^{7}$ CARrere, Claude, Barcelone, centre économique à l'époque des difficullés, 1380-1462, t. I, Paris-La Haye 1967, p. 278.

${ }^{8}$ HeErs, J., Le livre de comptes de Giovanni Piccamiglio..., op. cit., p. 15 , n. 3 et pp. 21-23. 
La galère est surtout un bâtiment de combat. Nos documents catalans l'appellent le plus souvent galera, ce qui tend à la distinguer de la galea marchande; le premier terme, en Catalogne, a supplanté le second au cours du xıvème siècle ${ }^{9}$, et cette évolution linguistique doit correspondre à la substitution du second type de bâtiment par le premier. Les Catalans eneffet se désinteressèrent, à la fin du xivème siècle, des galères marchandes, qui étaient relativement lourdes, difficiles à manoeuvrer et peu rentables en comparaison des nefs; ils imitaient en cela les Génois $^{10}$. Mais ces derniers, dans un tel choix, étaient mus surtout par une cause économique: leur monopole du trafic de l'alun qu'assuraient leurs nefs massives. Par contre le commerce catalan n'était pas déterminé par la prépondérance d'un produit pondéreux; l'abandon de la galère marchande dut avoir ici des raisons également militaires: il fallait être en mesure de tenir tête aux escadres génoises, composées de nefs et de galères rapides. Alphonse $V$ essayait d'égaler le tonnage élevé des nefs génoises, au grand dam des finances de la Couronne d'Aragon; on devrait alors tenter de vérifier s'il n'y eut pas une concurrence analogue en ce qui concernait les caractéristiques des navires catalans plus spécialement affectés aux transports commerciaux. Les données du problème se précisent quand on constate qu'au milieu du xvème siècle presque tout le commerce catalan vers le Méditerranée orientale s'effectuait sur des galères, ainsi que la majeure partie de ce commerce avec les Flandres". Les frais engagés par le Magnanime afin de disposer de nefs lourdes avaient donc bien certainement des motifs surtout stratégiques.

${ }^{9}$ Dufource, Ch. E., op. cit., pp. 36 et 39.

${ }^{10}$ HeERS, J. «Types de navires et spécialisation des trafics en Méditerranée à la fin du Moyen Age» (dans Le Navire et l'Economie maritime..., op. cit., p. 115). Au xvème siècle les Génois avaient abandonné les galères marchandes relativement lourdes et peu manoeuvrables dont ils se servaient encore au XIvème siècle (LANE, Frédéric C., «Tonnage Medieval and Modern», dans The Economic History Review, Zème série, vol. XVII, n. 2, 1964, pp. 219-231).

11 CARRERE, Claude, kLe droit d'ancrage et le mouvement du port de Barcelone au milieu du xvème siècle», op. cit., p. 96. 
Toutefois la difficulté de distinguer nettement entre navires marchands et navires de guerre continue d'entretenir une équivoque au sujet des galères catalanes. Il faudrait pouvoir les comparer de façon précise à celles de Venise, qui demeura fidèle à deux types de galères: la galera sottile pour le combat, longue, effilée, bien pourvue de rameurs professionnels en plus de l'unique voile latine, son atout principal étant la rapidité de manoeuvre; et la galera grossa pour le trafic, plus large, avec une chiourme réduite et une voilure plus développée ${ }^{12}$. Et si Gênes avait abandonné, vers la fin du xıvème siècle, la galère marchande (en génois galeazza, qui a donné le français galéasse), la galera sottile génoise coexistait avec la galera bastarda, bâtiment de combat plus lourd; dans l'ensemble, les galères génoises étaient plus rapides que celles de Venise, car elles étaient destinées, suivant la géopolitique génoise, à des opérations plus rapprochées ${ }^{13}$.

Le nombre des galères de guerre vénitiennes était à peine supérieur à celui des escadres génoises ou catalanes: Venise disposait en effet d'une dizaine de galères militaires en temps de paix et pouvait y ajouter, pendant une période de tension, une vingtaine d'unités de réserve tirées de l'Arsenal d'Etat. Mais ni Gênes ni la Couronne d'Aragon ne pouvaient apparemment fournir l'effort financier nécessaire pour entretenir en permanence une flotille de dix galères de guerre ${ }^{14}$.

La galera catalane ordinaire jaugeait 200 à 500 botes $^{15}$. Si l'on se base sur les équivalences approximatives que nous avons discutées précédemment, la documentation dont nous disposons pour les xıvème et xvème siècles peut fournir les estimations suivants:

Venise: galère sottile: environ 130 tonneaux galère grossa: environ 240 tonneaux

12 GILLE, Paul, «Transports maritimes et fluviaux», dans l'Histoire générale des techniques, dirigée par Maurice Daumas, t. II, Paris 1965, p. 394 et 404-405.

13 HeErs, Jacques, Gênes au Xvème siècle, Paris 1961, pp. 270-271.

14 Braunstein, Philippe et Delort, Robert, Venise, portrait historique d'une cité, Paris 1971, p. 109.

15 Carrere, Claude, loc, cit. 
Gênes:

galeazza: environ 130 tonneaux galère sottile: environ 100 tonneaux

Barcelone: galère:

galère bastarda: environ 130 tonneaux environ 300 à

800 tonneaux

On retrouve bien ici la politique génoise, qui allégeait les galères - avant tout navires de combat - en confiant le trafic commercial aux nefs lourdes. Mais nous restons dans l'incertitude à propos des galères catalanes, car la marge d'estimation demeure ici trop large pour bâtir des conclusions. Tout au plus peut-on constater que ces galères catalanes sont plus lourdes que leurs rivales génoises, sans doute parce qu'elles servaient davantage pour le commerce; elles sont aussi plus lourdes que les galères marchandes vénitiennes: peut-être est-ce parce que les Vénitiens s'en sont remis de plus en plus, pour le transport commercial, aux coques et aux caraques? ${ }^{16}$ Alphonse V, là encore, fut gêné par ce handicap du poids: il n'avait pas de galères aussi rapides que celles des Génois, tout en étant forcé d'essayer d'égaler ceux-ci pour les nefs, afin d'affronter les escadres génoises pour appuyer des opérations terrestres à longue portée, sans se disperser dans des raids de course.

Les Catalans avaient aussi des galères «armées», sans qu'on voie bien la différence avec des galères proprement commerciales. La flotte envoyée par Alphonse $\mathrm{V}$ pour accompagner un ambassadeur à Gênes, en 1421, porte 40 arbalétriers par galère: simple prudence afin de protéger une mission pacifique. Mais en 1423 des galères dites «armées» sont envoyées par le Magnanime à Majorque et portent des «gens d'armes» c'est-à-dire des cavaliers. Fin juin 1439, la "grosse galère neuvé» qui va de Barcelone à Naples emporte 300 arbalétriers. Ce dernier chiffre parait considérable en comparaison de l'effectif de l'équipage, qui pou-

${ }^{16}$ Les «vaisseaux ronds» (coques, caraques, de tonnage refativement moyen) tiennent la première place dans les constructions navales à Venise au début du xvème siècle (LANE, F.C., Navires et constructeurs à Venise pendant la Renaissance, Paris 1965, p. 96). 
vait comprendre au minimun un peu plus de 100 rameurs et au maximum $280^{17}$. Rappelons qu'une nef portait 500 hommes au maximum: il est vrai que sa marche à la voile nécessitait un équipage plus réduit que la galère. De toute façon la galère catalane armée du xvème siècle servait de transport de troupes en même temps que d'instrument de combat, à la différence de sa rivale génoise: et ceci demeure toujours en rapport avec la stratégie terrestre d'Alphonse V. Précisons que la galère "grosso» marchait mieux sous voiles qu'à la rame; et dans la première moitié du xvème siècle elle semble avoir eu deux mâts: un mât central et un mât de misaine plus gros que le grand mât: c'est pourquoi le nombre de rames y était réduit et l'on y disposait donc d'un espace plus grand pour la cargaison ${ }^{18}$.

Les galères transportaient-elles aussi les chevaux? Nos documents ne permettent pas de l'affirmer; d'ailleurs on ne voit guère où ils auraient pu trouver place. Toutefois des galéasses auraient parfois chargé des chevaux pendant les Croisades, entrés dans leur cale grâce à des «huis» recloués ensuite et calfatés ${ }^{19}$. C'est sans doute le même type de navire que l'uxer (huissier) catalan des xivème et xvème siècles, plus gros que la galère, avec davantage de rames, apte à porter chevaux et machines de guerre ${ }^{20}$. Ce type de "galère-hussier» fut d'un emploi fréquent dans les ports de Catalogne et du Midi de la France aux xmème et xivème siècles ${ }^{21}$. A la même époque, dans les flottes italiennes, les

17 Carrere, Claude, loc. cit. DufourcQ, op. cit., p. 51.

Les galères vénitiennes au Xvème siècle avaient de 150 à 190 rameurs; celles de Gênes (en 1454 exactement) 166 (HEERs, J., op. cit. p. 270 et ForTI, Umberto, Storia della tecnica dal Medioevo al Rinascimento, Florence 1957, pp. 296-299).

${ }^{18}$ History of Seafaring based on Underwater Archeology, éd. BASs, George F., New York 1972, p. 211.

${ }^{19}$ Merrien, Jean, La vie quotidienne des marins au Moyen Age: des Vikings aux galères, Paris 1969, pp. 202-203.

${ }^{20}$ SALAS, Don Francisco Javier de, «Hallazgo de la nave y galera del siglo XıI en el notable Códice de las Cántigas», dans Museo Español de Antigüedades, t. VI, Madrid 1875, p. 47-58.

${ }^{21}$ JAL, Augustin, Archéologie navale, t. 1, Paris 1840, p. 444 en notes. 
«huissiers» étaient des nefs: ici encore on constate l'archaïsme de la marine catalane en matière de technique.

On a cru, sur la foi des sources des xivème, xvème, et du début du xvıème siècles, que les galères catalanes portaient également des bombardes. Mais manifestement ces sources confondent ici les galères avec les nefs. On peut donc affirmer seulement qu'à partir de 1369 des nefs catalanes portèrent des bombardes $^{22}$.

Le terme latin triremis est employé parfois pour la galère: nos documents en font mention plusieurs fois à propos de sept galères du Magnanime en 1445 et de plusieurs autres en 1446, dont trois génoises. Il peut s'agir d'une équivalence purement littéraire: simple réminiscence humaniste pour désigner un navire plus grand qu'à l'ordinaire ${ }^{23}$. On a voulu peut-être aussi désigner ainsi le nombre de rameurs par rame, soit ici trois; nous avons peine à penser en effet qu'il s'agisse de trois rangs de rames superposées: ce dispositif aurait rendu la manoeuvre de la galère extrêmement difficile, car les rames étaient longues et lourdes ${ }^{24}$. La trirème aurait donc été une galère plus étroite, plus efuilée que le type ordinaire, qui comportait cinq hommes par aviron. Mais ceci l'aurait-elle rendue plus apte au combat, puisque le nombre des rameurs était moindre? Et il se peut au contraire que la trirème ait été plus large qu'une galère ordinaire, à l'image des galères mixtes vénitiennes qui effectuaient le transport des marchandises et celui des pèlerins de Terre Sainte ${ }^{25}$. Dans ce dernier cas, la trirème catalane aurait pu servir à porter des troupes, des vivres,

22 Cf. supra n. 4.

${ }^{23}$ Morrison, J.S., The Greek Triremes, dans The Mariner's Mirror, vol. XXVII, n. 1, janvier 1941, p. 19; JAL, A., op. cit., t. 1, p. 35.

${ }^{24}$ ForTt, loc. cit. MERRIEN J., op. cit., p. 223, compte $0,40 \mathrm{~m}$ par rameur sur une galère: à 3 hommes par rame, cela donnait pour le navire une largeur d'environ $3 \mathrm{~m}$, alors que d'ordinaire les galères avaient de 4,30 à $6 \mathrm{~m}$ de large, avec 5 hommes par aviron pour 25 à 27 bancs sur chaque bord (HEERs, loc. cit.). Il est vrai que ces 5 rameurs pouvaient tenir seulement la place de 3 , s'il y en avait 3 d'un côté de la rame et 2 en face, poussant et tirant symétriquement (GILLE, op. cit., p. 394).

25 Ces galères étaient les plus larges, avec $6,50 \mathrm{~m}$ Mais le francbord étant plus élevé, on avait réduit à $9 \mathrm{~m}$ la longeur de la rame, ordinairement de près 
du matériel: type de bâtiment moins coûteux à construire qu'une lourde nef, et dont il existait assez d'exemplaires en Catalogne pour qu'il fut assez facile d'en acheter, d'en louer ou d'en réquisitionner.

Une donnée documentaire complique encore le problème. Le 20 janvier 1422 Alphonse V, écrivant de Naples au trésorier de son royaume de Sicile, lui ordonne de préparer du matériel naval à livrer au patron d'une galea - le document est en latin-que le roi veut envoyer en Calabre: le trésorier devra d'abord rassembler 120 rames et les faire poser dans les tolets ${ }^{26}$. A première vue, cette galère aurait donc eu 60 bancs: nombre excessif pour l'époque. Y avait-il donc effectivement sur ce bâtiment, pour chaque banc, plusieurs avirons échelonnés, de longueur différente? N'était-ce pas le système italien a terzaruolo, toujours en usage au xvème siècle, où l'on croit comprendre que trois rameurs par banc, pour 26 bancs par bord, avaient chacun une rame, soit 156 rames au total? ${ }^{27}$ Mais d'après ce système il y aurait eu seulement 20 bancs par bord, ce qui est relativement peu: les galères catalanes avaient ordinairement 16 à 28 bancs de rameurs, chiffres voisins de ceux des galères italiennes du xvème siècle. Et l'ensemble détonnerait avec le terme de galea (et non galera) qui demeure employé, dans ce début du xvème siècle, pour une grande galère. Il vaudrait mieux supposer deux avirons par banc, ce qui donne 30 bancs: c'est beaucoup, mais cela cadre davantage avec le mot galea.

Toutefois on peut aussi concevoir que cette galère de janvier 1422 ait comporté plusieurs étages de rames. Un tel bâtiment «birème» aurait alors compté 30 rangs par étage et par bord, et une «trirème» 15 rangs de même. Et l'on retrouve ici le problème posé par le terme triremis. A moins que l'on n'émetre une autre hypothèse: les rames devaient être toutes essayées dans les

de $12 \mathrm{~m}$ : soit 2 à 3 hommes par aviron; la vitesse était moindre, mais l'espace disponible était plus grand (GILLE, op. cit., p. 405).

${ }^{26}$ Barcelone, Archives de la Couronne d'Aragon, registre de chancellerie 2807, A IV Comune Sicilia, f. 19.

27 ForI, loc, cit. MERRIEN, op. cit., p. 197. 
tolets, mais un certain nombre devait être ensuite mis en réserve sur la galère, comme rechange: inutile alors de supposer plusieurs étages de bancs.

Quoi qu'il en soit, chaque hypothèse appelle une conclusion commune. Ou bien la trirème catalane est à étage unique, avec trois hommes par aviron: sa force de propulsion se trouve alors inférieure à celle des galères ordinaires à cinq hommes par rame. Ou bien, toujours avec trois hommes par aviron, la trirème est plus large qu'une galère ordinaire et sa vitesse en est encore amoindrie, d'autant plus que l'augmentation de l'espace disponible était peut-être utilisé pour charger des troupes, des vivres ou du matériel. Ou encore il y avait plusieurs rames par banc; ou enfin plusieurs étages avec un banc sur chacun d'eux: dans ces deux derniers cas la construction du navire, son équipement, sa maoneuvre étaient plus complexes, et certainement plus coûteux et plus aléatoires. On a donc l'impression d'un certain archaïsme technique en ce qui concerne les galères catalanes du xvème siècle.

Mais cet archaïsme se prolongea-t-il longtemps? Nous manquons sur ce point des renseignements nécessaires. Les trirèmes de 1445 pourraient servir de jalon dans une séric qui reste à reconstituer, si tant est que l'on puisse apporter des précisions sur le type exact de ces «trirèmes»: et encore faudrait-il savoir si elles avaient été construites en Italie ou en Catalogne. Il est un autre point qui confirme l'obscurité de la documentation: nous ne savons pas à partir de quand, sur les nefs et les galères catalanes, le gouvernail-aviron de type antique fut remplacé par le gouvernail d'étambot. Aucun auteur, à notre connaissance, ne parait s'être préoccupé de façon précise de ce problème, dont la solution permettrait de mieux évaluer les capacités manoeuvrières des bâtiments catalans, à condition de pouvoir effectuer des comparaisons avec l'évolution des autres marines méditerranéennes dans le même domaine. Nous savons seulement que, sur les galères italiennes et castillanes, le gouvernail-aviron et le gouvernail d'étambot furent tous deux concuremment en usage au xıvème siècle, tandis qu'au xvème le gouvernail-aviron se trouvait mis en réserve pour suppléer le gouvernail d'étambot dans 
les manoeuvres difficiles; il en fut de même sur les nefs italiennes jusqu'au xvème siècle ${ }^{28}$.

A supposer que cet archaïsme naval des Catalans se soit prolongé, ce fait concorderait avec la stratégie maritime d'Alphonse V, qui misait pour les nefs sur le poids, les bombardes, les soldats embarqués, et pour les galères sur la vitesse de pointe: alors que ces dernières, en dehors des combats, servaient aussi pour les transports militaires. Alphonse V aurait ainsi négligé la guerre de course afin de s'interesser avant tout au support maritime d'opérations terrestres à longue portée. La marine aurait été pour le Magnanime un instrument logistique bien plus qu'une force indépendante et prépondérante: au contraire des Génois, qui assurèrent de la sorte leur victoire à Ponza, sur les côtes napolitaines, le 5 août 1435 .

Une variante de la galère, en plus petit, c'est la galeota (ou galeotta). Alphonse V en envoie une à Gênes en compagnie de deux galères en octobre 1446, afin d'affronter les navires de Benedetto Doria. On doit noter que la galiote était appelée biremus, ce qui pose les mêmes problèmes que pour la galère «trirème». A propos des dimensions de la galiote, on ne distingue pas ici d'indications précises, excepté dans un traité conclu par Ancône avec Venise en 1446, où la première cité promet à la deuxième de l'aider avec deux galiotes de 16 bancs chacune; on voit aussi le duc de Milan Philippe-Marie Visconti employer des galiotes afin de combattre sur le lac de Garde en 1440: deux de 22 bancs et trois de 15 bancs. La galiote est donc une petite galère, apte au transport avec ses 140 botes en général ${ }^{29}$, mais plus convenable encore pour le combat, car elle suppléait au fait que la galère était plus gênée que la nef par mauvais temps, à cause de la faible hauteur du franc bord de la galère ${ }^{30}$. En ce qui concerne l'importance numérique relative des galiotes dans les engagements navals, complétons nos informations en rappelant que

28 JAL A., op. cit., t. 2, p. 83, Id., Glossaire nautique, Paris 1848: articles Alla navaresca (t. I, p. 101, col. 1), Governal (vieux français: t. I, p. 798 , col. 1), Timo, Timon, Timone (t. 2, p. 145LK, col. 2, p. 1454, col. 1).

29 CARrere Claude, «Le droit d'ancrage...», op. cit., p. 96.

${ }^{30}$ GIL.E, op. cil., p. 404. 
René d'Anjou parvint à Naples en 1438 avec 12 galères et 4 galiotes. On constate ainsi que la proportion moyenne des galiotes aux galères était de 1 pour 2 ou 3 .

\section{Autres types de navires}

La détermination du type de bâtiment s'est avérée difficile en ce qui concerne les galères. Elle l'est encore plus avec la barca. Nos documents fournissent ici des renseignements bien fragmentaires? L'amiral catalan d'Alphonse V a beaucoup de barche dans l'Adriatique, en plus de six galères, en 1445: était-ce pour préparer un débarquement manqué? Les six galères ont en effet attaqué vainement le port de Fermo et bombardé celui de Pesaro. Les «barques» de cette escadre, en tous cas, ont servi pour transporter des troupes sur 40 ou 60 kilomètres le long de cette côte italienne de l'Adriatique. Et une «barque» catalane fut prise en chasse deux jours et deux nuits par une lourde nef génoise en 1438: la barca, tout en pouvant servir de transport -comme la galère catalane - était donc un bâtiment relativement léger.

Par comparaison, nous savons qu'en 1446 une «barque» italienne, montée par un patron, quatre marins et un passager, fut prise dans l'Adriatique par une fusta corsaire de François Sforza: faible équipage, donc navire petit; mais l'incertitude oủ nous sommes des caractéristiques de la fusta ne nous permet pas de mesurer en proportion les insuffisances de la barca. L'équipage était encore inférieur - un patron et trois marins - pour une «barque» de Marseille capturée par une galère catalane au large de Gênes en 1444.

On peut toutefois pousser la comparaison grâce à un document barcelonais: c'est une information anonyme et non datée, que l'éditeur place en 1435, mais qu'il convient de reporter plutôt en 1436, quand les troupes du duc de Milan dévastaient le territoire de Gênes qui venait de rejeter la domination du Visconti" ${ }^{31}$. L'informateur signale que les Génois meurent de

${ }^{31}$ Madurelt. Marimon, José Maria, Mensajeros barceloneses en la corte 
faim, car ils ne peuvent se ravitailler qu'avec ce que leur apportent leurs barcas; et si leurs ennemis disposaient de fustas, préciset-il, Gênes serait prise. Ces indications sont plausibles; après la révolte génoise, Philippe-Marie Visconti n'avait plus de flotta: Alphonse V, affaibli par sa défaite à Ponza, n'avait pas les moyens de bloquer Gênes; de leur côté, les Génois avaient besoin de leurs nefs pour la guerre contre les Catalans, et ne pouvaient alors s'en servir pour des tâches de ravitaillement. Les «barques» sont donc ici des bâtiments à but commercial, apparemment inférieures aux "fustes» au point de vue militaire, ce qui confirme nos renseignements précédents. Confirmation reforcée par d'autres documents qui nous parlent de deux «barques» toscanes pillées par deux brigantins sardes en septembre 1422, et dont l'une d'elles, qualifiée de «grande» (en latin), transportait du blé. Nous touchons ici probablement le maximum du tonnage que pouvait atteindre une «barque», car elle pouvait alors servir d'escorteur: en juin 1439, Venise arma en guerre trois "grandes barques" (encore en latin) pour escorter un transport de vin qui lui arrivait des Marches. On peut donc penser que la «barque» est un petit caboteur.

On sait d'ailleurs que les «barques» génoises ne dépassent jamais 500 cantari, soit environ 24 tonnes $^{32}$. Et ces «barques» pouvaient également servir à la fois de chaloupes et de bâtiments de transbordement pour les nefs génoises mouillées au large de ports ne disposant pas d'appontements suffisants: une nef génoise portait en effet généralement une «barquen de 60 rames $^{33}$. Le fait que cette embarcation n'est qualifiée ni de biremis ni de triremis nous autorise à penser qu'elle avait un rameur par rame, soit trente bancs de nage: chiffre qui l'apparante à une galère. Mais on imagine mal une galère portée par une nef, même si le tonnage de cette dernière était de deux à cinq fois supérieur

de Nápoles de Alfonso V de Aragón, 1435-1458, Barcelone 1963, p. 300, document 2362.

${ }^{32}$ HeERs Jacques, op. cit., p. 280. Rappelons que le cantar génois valait 47,6 kilos.

${ }^{33}$ Id., ibid., p. 273. 
au tonnage de celle-là, comme l'analyse pratiquée plus haut nous permet de l'estimer. Nous pouvons donc supposer qu'une partie de ces avirons était prévue comme rames de rechange: hypothèse que nous av́ons déjà formulée concernant une galère d'Alphonse V. La «barque» à bord de la nef génoise aurait eu dès lors en moyenne quinze bancs: avec un seul rameur par banc, cela représente une chaloupe assez forte, apte aux opérations de transbordement et d'un débarquement important.

Mais les «barques» dont nous venons de parler sont italiennes, et nos documents ne nous permettent pas d'appliquer nos conclusions sans réservé aux «barques» catalanes. Ainsi les chaloupes sont mieux déterminées sur les bâtiments catalans importants, puisqu'elles portent le nom de squiffes, au moins au xrvème siècle ${ }^{34}$. Et vers le milieu du xvème siècle à Barcelone, il y avait des barquetres équivalant plus ou moins à des esquifs: c'était des sortes de chaloupes à un mât, non pontées, de 8 à $11 \mathrm{~m}$ de long, et jaugeant au maximum $10 \mathrm{botes}$, soit environ 4 tonnes de port; elles pratíquaient le cabotage entre Valence, Barcelone et le Midi de la France, et se livraient également à la pêche côtière, activité que ce même type d'embarcation continue de nos jours"s.

Et l'on ne sait pas si l'utilisation des «barques» catalanes était aussi diversifiée que celle des italiennes. On peut le supposer, car dès le xivème siècle le mot barca (ou barcha), chez les Catalans, est beaucoup plus employé que le terme fusta, qui s'appliquait pourtant à toute espèce de navire au xıvème siècle. Mais on risque alors de confondre barca et leny: or la première n'était probablement qu'une catégorie moyenne du second. Toutefois on peut se demander si le tonnage minimum de la barca n'avait pas rejoint celui du leny au xvème siècle: au xivène en effet celle-là ne comportait pas moins de 10 à 15 hommes d'équipage, tandis que celui-ci pouvait être monté par 4 ou 5 matelots seulement, ce dernier cas étant celui de «barques» italienne et marseillaise navigant en $1444-1446^{36}$. On doit noter que le ter-

34 DUfourcQ, Ch.-Emm., op. cit., p. 39.

35 CARRERE, op. cit., pp. 90-91.

36 DufourCQ, op. cit., p. 38. 
me leny n'apparait pas dans nos documents du xvème siècle: comme ceux-ci concernent essentiellemente les côtes italiennes, peut-être ce terme typiquement catalan s'est-il trouvé supplanté, dans ce secteur géographique, par le mot barca, qui est à la fois italien et catalan? Ou bien l'utilisation des «barques» par les Catalans fut-elle réellement limitée? Ce serait alors un nouvel exemple d'archaïsme technique en ce qui concerne les flottes d'Alphonse V. Et pourtant ce dernier devait avoir bessoin de navires de débarquement et de transbordement, vu l'orientation générale de ses opérations destinées à prendre pied sur la terre italienne de façon stable et pour longtemps. Mais on peut supposer qu'il chargeait au maximum ses approvisionnements sur ses nefs et sur ses galères, au risque de compromettre leurs capacités manoeuvrières, par souci d'économie. Du reste, une fois maitre d'un port important, il pouvait trouver des «barques» sur place et faire approcher ses navires lourds au plus près de la côte, peutêtre même les mettre à quai.

Ces hypothèses nous semblent confirmées par les renseignements que donne la documentation sur les navires fréquentant le port de Barcelone au milieu du xvème siècle ${ }^{37}$. On y voit des lenys et des barques, sous bâtiments pontés, donc navires de charge. En considérant les lenys proprement dits, on relève des tonnages de 20 à 80 botes, soit 18 à 20 tonnes. Pour la barca normale, on va de 20 à 60 botes, soit 8 à 24 tonnes; avec une moyenne de 30 botes, soit 12 tonnes. Il n'y a pas encore là de distinction bien précise avec le leny. Mais certaines «barques» sont mieux caractérisées, car elles jaugent entre 100 et 200 botes: 40 à 80 tonnes de port; on retrouve là les «grandes barques» italiennes: en effet ces embarcations catalanes, en plus du cabotage, assurent une partie du trafic entre la Catalogne, la Sardaigne et l'Afrique du Nord. Les plus grosses vont jusqu'en Italie et peuvent même affronter l'Atlantique, y compris en hiver. Nous dépassons ici les comparaisons incertaines entre les échelles de tonnage des lenys et des barcas: les "grandes barques» catalanes s'apparentent manifestement aux cocas, transporteurs catalans à voile,

${ }^{37}$ CARRERE, op. cit., pp. 90-93. 
mis au point au xivème siècle sur le modèle de la kogge (ou «coque» en français) flamande, effectivement capables de naviguer sur l'Océan comme en Méditerranée ${ }^{38}$. Mais la comparaison s'arrête là: la «coque» était de forme ronde, et nous ne connaissons pas le dessin de la «barque». Au reste les Catalans, séparés de l'Océan par la Castille, avaient très peu de cocas.

Etant donnée cette échelle approximative du tonnage des «barques» et leur caractère fréquent de transbordeurs, leur propulsion devait être assurée principalement à la rame. Mais nos documents ne précisent rien à ce sujet, si ce n'est que les «petites barques» (barquetes) barcelonaises avaient un mât, donc au moins une voile. Nos documents ne précisent rien non plus sur ce point pour la fusta, dont nous avons déjà quelque peu parlé. Nous avons vu qu'en catalan, pendant le xıvème siècle, ce terme était resté vague et relativement rare. La supériorité militaire de la fusta sur la barca nous a été confirmée par les prises d'une "fuste» de François Sforza dans l'Adriatique en 1446: 3 «barques») et un barcoso (grande barque employée surtout comme caboteur de transport); cette «fuste», il est vrai, est qualifiée de «corsaire»: mais on ne sait si cette epithète caractérise uniquement une action momentanée de ce type de bâtiment, ou un armement complémentaire de celui-ci destiné à la course. Le caractère militaire de la «fuste» nous est cependant confirmé dans l'annonce transmise en Catalogne par Alphonse V, en mars 1436, de l'imminence d'une attaque génoise contre les iles de la Couronne d'Aragon qui sera menée par 5 nefs «et d'autres fustes ${ }^{39}$. Ce dernier terme reste encore ici peu déterminé, mais il est plus distinct dans un document de novembre 1445: Alphonse V y annonce son intention de rallier toutes les «fustes» qui croisent sur les côtes de ses possessions espagnoles, car il en a données aux Génois, ce qui fait qu'il n'en a pas assez pour combattre Venise et Florence. Jal donne seulement deux précisions sur la fusta: bâtiment à rames, avec 15 bancs au maximum ${ }^{40}$ : embarcation plus petite que la "grande barque» par

${ }^{38}$ DUFOURCQ, op. cit., p. 41.

39 MadURELL. MARIMON, op. cit., pp. 18-21.

$40 \mathrm{JAL}$, Glossaire nautique, cit., art. Fusta (t. I, p. 726). 
conséquent, et plus maniable que celle-ci, d'où la supériorité de la «fuste» sur le plan militaire.

Nous avons recueilli quelques mentions d'un autre type de navire: le «brigantin» (en italien brigantino ou bergantino; en catalan barquanti ou bergant $i$ ). Ces mentions retracent des actions de pillage exercées par des brigantins sardes contre des "barques» toscanes (dont une "grande») en septembre 1422. Et René d'Anjou avait deux brigantins dans la flotille qu'il amena de Gênes à Naples en avril 1438, en plus des douze galères et quatre galiotes composant cette formation. Nous voyons qu'il devait s'agir de bâtiments auxiliaires ayant les mêmes qualités militaires que la fusta. Notons qu'il n'est pas fait mention, dans nos documents, de brigantins italiens: la terminologie catalane serait donc plus ancienne; en tous cas, elle montre bien qu'il s'agit d'une sorte de «barque», certainement plus rapide que la «barque» ordinaire: il n'est pas question d'une origine étymologique venant de «brigand». De fait, le brigantin barcelonais était un bâtiment à rames, jaugeant le plus souvent 40 botes, soit environ 16 tonnes de port ${ }^{41}$. Mais il reçut des voiles au xvème siècle $\mathrm{e}^{42}$ et ses dimensions augmentèrent alors: de 8 à 12 bancs par bord -à un rameur par banc-il en vint à compter 16 à 24 rames, avec deux voiles latines; mais ces transformations ne s'opérèrent que de façon lente, et probablement dans les flottes italiennes ${ }^{43}$.

Il nous reste à relever la mention d'un balanorius armatus (armé en guerre), en janvier 1420, qui réussit à piller une nef génoise entre Gênes et Aigues-Mortes. D'autres indications sont données par Jal ${ }^{44}$ : une lettre des Barcelonais d'août 1423 qui parle de baleners cum velas, et un extrait d'une chronique génoise

${ }^{41}$ Carrere, op. cit., p. 93.

42 JAL, op. cit., art. Bergantí (t. I, p. 342, col. 1).

${ }^{43}$ FEA, Leonardo, art. Nave dans I'Enciclopedia Italiana Treccani, t. XXIV, Rome 1934, p. 347, col. 2.

44 JAL, op. cit., art. Balaneria (t. I, p. 221, col. 2) et art. Baleinier 1 (id., pp. 223-224): on trouve effectivement au xvème sièclc, en catalan, les formes balaner et balane. 
contemporaine qui signale en 1423 la sortie de Gênes d'une escadre destinée à secourir la reine de Naples Jeanne II: cette formation comprenait, à côté de 9 grandes nefs génoises, 4 navires plus petits dont 2 «baleiniers», ceux-ci portant de 200 à 220 hommes chacun. Il est surprenant que ce chroniqueur caractérise le baleinier comme très petit (admodum parvis), alors qu'il pouvait porter presqu'autant d'hommes qu'une nef ou une galère. On peut penser que les formes du baleinier étaient plus fines que celles de la nef, à laquelle il était apparenté, comme navire à voiles, par son dessin général. Il faudrait, pour une appréciation plus juste, être capable d'estimer le tonnage d'un baleinier: mais nos documents restent muets sur ce point.

L'on sait pourtant que ces baleiniers étaient employés pour la guerre par les Catalans et les Génois depuis le milieu du xıvème siècle. Capmany est allé même jusqu'à leur attribuer un tonnage de 4.000 botes, soit 1.600 tonnes de port environ: mais on reste sceptique en sachant, d'après Jal, que l'érudit espagnol a confondu ici des baleiniers avec des nefs ${ }^{45}$; et son estimation se révèle discutable même pour une grosse nef génoise: elle est absolument contradictoire avec le témoignage du chroniqueur génois cité par Capmany lui-même. On peut penser, dans un juste milieu, que le baleinier, sans avoir un tonnage très faible pour l'époque, était utilisé pour la guerre et particulièrement pour la course. Il faudrait chercher si cet emploi s'expliquait par les formes et le gréement de ce type de navire. Avait-il un rapport avec la pêche à la baleine pratiquée dès le Moyen Age dans l'Atlantique? C'est possible: Heers nous apprend que vers 1460 des Basques et des Portugais introduisirent des baleneros en Méditerranée pour transporter un trafic de faible tonnage, et que les Génois, peu après, suivirent cet exemple en utilisant des baleiniers pour le transport des produits de luxe faiblement pondéreux (épices, soie); la prise des mines d'alun génoises en Asie Mineure compromettait en effet la rentabilité des grosses nefs que les Génois avaient spécialisées dans le transport de l'alun, matière de poids ${ }^{46}$.

45 CAPMANY, op. cit., t. I, Madrid 1779, p. 41.

${ }^{46}$ HeERS, Jacques, Types de navires et spécialisation des trafics... cit, p. 117. 


\section{Conclusion}

Nous avons essayé de distinguer au mieux les types de navires mentionnés dans notre documentation. Mais on a pu mesurer les insuffisances de celle-ci. Point de précisions satisfaisantes sur le tonnage, soit en jauge soit en port, ni sur les formes dẹs bâtiments, ni sur leur mode de propulsion, ni sur l'importance et la composition des équipages, non plus que sur le genre d'utilisation des navires. Le doute continue de régner en particulier sur la disposition des rameurs dans les galères. La terminologie demeure toujours incertaine, aussi bien en latin qu'en italien ou en catalan. Les unités de mesure restent mal estimées. Ces difficultés proviennent probablement du fait que nos documents sont avant tout des correspondances d'ordre diplomatique ou militaire, informant au plus vite, et donc de façon brève, sur les mouvements des unités navales, afin de prendre des dispositions défensives ou offensives, ou encore pour demander l'argent nécessaire à préparer des renforts.

On a bien également quelques chroniques: elles ne comblent guère les lacunes; on ne peut attendre de leurs auteurs, qui étaient des terriens, que des récits évènementiels trop succincts ou trop littéraires. Les instruments de travail linguistiques - Jal ancien et nouveau, Du Cange - apportent peu de chose pour le xvème siècle. En matière iconographique, nous n'avons rien trouvé sur le présent sujet. Nous pensons qu'une recherche plus approfondie devrait s'orienter vers des documents comptables: états préparatoires et bilans des frais de préparation des flottes et de leur entretien.

On s'explique notre hésitation devant une tentative de classement des bâtiments répertoriés. Beaucoup servent indifféremment pour le commerce et pour la guerre, et sans doute aussi pour la pêche. Un grand nombre marche aussi bien à la voile qu'à la rame, en utilisant probablement les deux en même temps dans certaines circonstances. On aura constaté que nous avons adopté le modeste parti d'étudier ces navires suivant la fréquence et l'importance de leurs mentions dans nos documents en ordre décroissant. Là encore il faut être prudent: ce n'est pas parce 
que des bâtiments de moindre tonnage, comme les «barques», sont exposés après les nefs et les galères, que le nombre total des premiers peut être supposé comme inférieur à celui des secondes à une date donnée, dans une flotte donnée; le contraire est même probable: en effet les unités de fort tonnage coûtaient sensiblement plus cher et leur construction durait plus longtemps.

Nous pouvons cependant dégager quelques conclusions. Les bâtiments les plus importants, pour la guerre comme pour le commerce, sont les nefs et les galères. Les nefs sont plus grosses, surtout celles des Génois, ce qui ne les empêche pas de manoeuvrer mieux que les nefs catalanes. Les galères sont plus rapides au combat: c'est pourquoi Génois et Catalans les utilisèrent de plus en plus pour la guerre, en escorte ou en appui des nefs. Et chez les Catalans la galère servait aussi de transport de troupes, ce qui contribuait à donner à la construction navale catalane un caractère relativement archaïque.

On voit que, si le poids des nefs armées en guerre constituait un elément de succés maritime, la rapidité de manoeuvre était également un atout considéré comme essentiel. On s'explique ainsi la spécialisation des galiotes, petites galères de combat qui servaient accessoirement de transports, ainsi que les «barques» les plus petites. La recherche de la vitesse est également sensible avec l'emploi de navires corsaires tels que les «fustes», les «brigantins» et les «baleiniers». Au contraire il est impossible de trouver un caractère commun pour la catégorie des «barques»), très largement ouverte en échelles de tonnage et comprenant des caboteurs marchands, des escorteurs, des bâtiments de transbordement, des chaloupes; il est à présumer qu'une même «barque» pouvait être apte à plusieurs de ces utilisations suivant les circonstances.

De toute façon la variété des types, des tonnages et des modes d'emploi se révèle assez étendue dans les flottes chrétiennes de la Méditerranée occidentale et de l'Adriatique. Et nous avons noté même une tendance à la spécialisation dans le domaine de la guerre de course. Il faut relever cependant que ces deux aspects sont plus marqués pour les flottes italiennes que pour celles du la Couronne d'Aragon. C'est pourquoi l'on ne peut s'empêcher 
de penser que la construction navante catalane accusait alors un certain retard, au moins au point de vue technique, sur celle des grands ports italiens. Il est possible qu'Alphonse V ait voulu concentrer ses moyens navals afin de réaliser des économies que lui demandaient sans trêve ses principaux baileurs de fonds, les bourgeois de Barcelone, et pour chercher en même temps à forcer la décision politique et militaire qui lui tenait le plus à coeur: la victoire sur les Génois, par le moyen de la conquête du royaume de Naples à partir de 1420. Mais le raisonnement peut être inversé: le roi concentrait ses moyens à cause de l'infériorité d'ensemble de ceux-ci; la possession de l'Italie méridionale devait servir à compenser cette infériorité: car en plus de l'importance de sa position stratégique, cette région pouvait fournir un complexe varié de ressources en matière d'armement maritime.

Il semble que l'évolution de l'économie générale ait joué de manière importante sur les politiques navales du Magnanime et de ses adversaires génois. Les uns et les autres évitèrent le plus possible les batailles en ligne et les blocus, à cause du coût très élevé des moyens nécessaires à ces actions. Du point de vue militaire, ils miserent surtout sur la vitesse de pointe des galères. Mais ces bâtiments exigeaient un équipage relativement nombreux et se prêtaient moins au grand commerce que les nefs. On peut penser que ces considérations financières incitèrent les belligérants à se servir de plus en plus de navires à la fois légers et rapides comme les fustes, les brigantins et les baleiniers, capables d'arraisonner les caboteurs et les embarcations de transbordement qui accompagnaient les grosses unités, l'accostage en eau peu profonde étant impossible pour ces dernières. Avant la conquête du royaume de Naples par Alphonse V, une telle évolution fut davantage favorable aux Génois, car leur ennemi devait acheminer vers l'Italie méridionale des renforts avec leur matériel de guerre et leur ravitaillement: les escadres catalanes étaient donc lourdement chargées, concentrant les bâtiments de fort et de moyen tonnage par mesure d'économie: ces escadres étaient alors vulnérables aux assauts des corsaires, dont les qualités manoeuvrières permettaient de disperser les convois et d'avoir raison des caboteurs plus ou moins isolés. Les Catalans, tout occupés 
à l'entreprise de Naples, durent souffrir de ce harcèlement sans pouvoir rendre coup pour coup: d'où leurs réticences pour appuyer cette expédition. Mais si celle-ci n'avait pas eu lieu, la flotte catalane, dont la composition technique paraissait tant soit peu retardataire, aurait-elle pu tenir tête aux Génois, réduire leurs prises et troubler au contraire leurs itinéraires commerciaux? Cet effort aurait nécessité sans doute une adaptation des bâtiments catalans à une vitesse plus grande au détriment du tonnage: et cette adaptation nécessitait donc à son tour une évolution du trafic, pour laquelle la Couronne d'Aragon semblait moins bien préparée que la République génoise, car celle-ci disposait d'une supériorité commerciale et financière.

La modernisation de la force navale d'Alphonse $V$ était d'autant plus difficile à réaliser que le manque de moyens financiers obligea le roi à limiter ses programmes de construction pour pouvoir mieux les étaler. C'était courir le risque de garder un retard croissant par rapport aux flottes adverses. Ce retard pouvait-il etre comblé par l'appoint des navires italiens capturés, réquisitionnés ou affrétés par le Magnanime? Cet appoint devait être insuffisant, car la flotte catalane avait subi des pertes sensibles dans la lutte à peu près continuelle qu'elle avait livrée, depuis la fin du xivème siècle, aux Génois et aux Vénitiens: lutte pour laquelle on a parlé de "saignée continuelle» se traduisant par une augmentation ininterrompue du prix des frêts et des assurances maritimes ${ }^{47}$. Le commerce catalan se trouvait plus vulnérable face à la concurrence croissante des flottes rivales, non seulement italiennes, mais océaniques: les Andalous et les Basques venaient désormais trafiquer — et pirater-en Méditerranée.

47 VICENS VIVES, Jaime, Evolución de la economía catalana durante la primera mitad del siglo $X V$, Palma de Majorque 1955, p. 14 (rapport présenté devant le IVème Congrès d'Histoire de la Couronne d'Aragon). 
FONDS DOCUMENTAIRES CONSULTÉS

(interessant l'Italie continentale et principalement les affaires maritimes).

Barcelone: Archives de la Couronne d'Aragon. Chancellerie royale d'Alphonse IV (V d'Aragon). Nous donnons successivement le nom de la série, les années concernées, les numéros des registres.

Varia (1413-1431) 2454; Comune (1414-1416) 2444, (1418-1425) $2467-$ 2473, (1441-1445) 2523-2528; Comunis sigilli secreti (1419-1426) 2568 2575; Gratiarum (1416-1429, et dernier acte 1456) 2588-2593; Officialium (1444-1448) 2598; Diversorum (1416-1453) 2609-2619; Sardinia (1447-1449: quelques documents sur les rapports avec Gênes) 1631; $\mathrm{Cu}$ rie (1416-1444) 2642-2650; Curiae (1435-1452) 2651-2658; Curiae sigilli secreti (1413-1420) 2542, (1413-1446) 2665-2678, 2685, 2690; Secretorum (1419-1458) 2694-2700; Itinerum (1442-1449) 2777-2779; Itinerum sigilli secreti (1420-1446) 2781-2786, 2794-2795; Comune Sicilia (14221424) 2807-2809; Curia Siciliae (1420-1427) 2888; Curia cancilleriae Napolis (1436-1440) 2900; Privilegiorum cancilleriae Napolis (1440-1449) 2902-2903; Instructionum (1440-1453) 2939; Instructionum et Albanorum (1416-1453) 2940.

Lugartenencia de la reyna doña Maria: Curias (1422-1424) 3168 (documents sur Naples: ff. 165-166).

Barcelone: Archives de la Cité.

Cartas comunas originals reebudes (1437-1444) voll. 9, 12-14. Barcelone: Archives de la Cathédrale.

Quelques Cartas reales. 